\title{
Molecular characterization of Glaesserella parasuis strains isolated from North America, Europe and Asia by serotyping PCR and LS-PCR
}

\author{
Nubia Macedo ${ }^{1 *}$ (D), Marcelo Gottschalk ${ }^{2}$, Katrin Strutzberg-Minder ${ }^{3}$, Chao Nguyen Van ${ }^{4,5}$, Lijun Zhang ${ }^{5}$, \\ Geng Zou ${ }^{5}$, Rui Zhou ${ }^{5}$, Thaire Marostica ${ }^{1,6}$, Maria Jose Clavijo ${ }^{1,7 \dagger}$, Alexander Tucker ${ }^{8 \dagger}$ and Virginia Aragon ${ }^{9,10 \dagger}$
}

\begin{abstract}
Glaesserella parasuis strains were characterized by serotyping PCR, vtaA virulence marker Leader Sequence (LS)-PCR, clinical significance, and geographic region. Overall, the serovars 4,5/12, 7, 1, and 13 were the most commonly detected. Serovars of greatest clinical relevance were systemic isolates that had a higher probability of being serovar $5 / 12,13$, or 7 . In comparison, pulmonary isolates had a higher likelihood of being serovars $2,4,7$, or 14 . Serovars $5 / 12$ and 13 have previously been considered disease-associated, but this study agrees with other recent studies showing that serovar 7 is indeed associated with systemic G. parasuis disease. Serovar 4 strains illustrated how isolates can have varying degrees of virulence and be obtained from pulmonary, systemic, or nasal sites. Serovars 8, 9, 15, and 10 were predominantly obtained from nasal samples, which indicates a limited clinical significance of these serovars. Additionally, most internal G. parasuis isolates were classified as virulent by LS-PCR and were disease-associated isolates, including serovars $1,2,4,5 / 12,7,13$, and 14. Isolates from the nasal cavity, including serovars 6, 9, 10, 11, and 15, were classified as non-virulent by LS-PCR. In conclusion, the distribution of G. parasuis serovars remains constant, with few serovars representing most of the strains isolated from affected pigs. Moreover, it was confirmed that the LS-PCR can be used for G. parasuis virulence prediction of field strains worldwide.
\end{abstract}

Keywords: Glaesserella parasuis, Serotyping, VtaA marker, LS-PCR

\section{Introduction}

Glaesserella parasuis is an important pathogen of swine and remains one of the most important bacterial causes of mortality in swine production worldwide. Clinical signs of infected pigs range from fever, respiratory signs, and swollen joints with lameness, to central nervous signs, and sudden death. Chronic infections are also associated with reduced growth rates and food conversion efficiencies. The main pathological findings include

\footnotetext{
*Correspondence: nubia@iastate.edu

${ }^{\dagger}$ Maria Jose Clavijo, Alexander Tucker and Virginia Aragon contributed equally to the work

${ }^{1}$ Veterinary Diagnostic Laboratory, lowa State University, Ames, IA, USA

Full list of author information is available at the end of the article
}

fibrinopurulent polyserositis, polyarthritis, and meningitis, commonly known as Glässer's disease [1].

Glässer's disease is considered one of the most prevalent swine bacterial infections worldwide [2]. Reliance on prophylactic or strategic medication to control this disease is an ongoing concern regarding the emergence of antimicrobial resistance [3-6]. Bacterins and a live attenuated vaccine are commercially available. However, G. parasuis is a highly diverse pathogen, with still poorly known cross-protection against disease-causing field strains $[7,8]$. In many countries, autogenous bacterins are also produced.

G. parasuis is also a commensal of the upper respiratory tract (URT) [9]. Disease outbreaks are associated with the introduction of novel G. parasuis strains into an

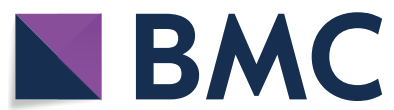

(c) The Author(s) 2021. This article is licensed under a Creative Commons Attribution 4.0 International License, which permits use, sharing, adaptation, distribution and reproduction in any medium or format, as long as you give appropriate credit to the original author(s) and the source, provide a link to the Creative Commons licence, and indicate if changes were made. The images or other third party material in this article are included in the article's Creative Commons licence, unless indicated otherwise in a credit line to the material. If material is not included in the article's Creative Commons licence and your intended use is not permitted by statutory regulation or exceeds the permitted use, you will need to obtain permission directly from the copyright holder. To view a copy of this licence, visit http://creativeco mmons.org/licenses/by/4.0/. The Creative Commons Public Domain Dedication waiver (http://creativecommons.org/publicdomain/ zero/1.0/) applies to the data made available in this article, unless otherwise stated in a credit line to the data. 
immunologically naïve pig population, typically through the mixing of pigs from different sources. Other predisposing factors include poor management, high stocking density, inadequate ventilation, and coinfections with other respiratory pathogens $[10,11]$.

Epidemiological investigation of G. parasuis-associated disease, and preventive health management, relies on identifying specific G. parasuis serovars colonizing and or causing illness. Fifteen G. parasuis serovars that differ in virulence have been described $[12,13]$. However, healthy pigs can carry serovars commonly isolated from systemic sites in the URT, alongside isolates of serovars rarely identified in clinical disease $[14,15]$.

This broad diversity within the G. parasuis species in the field requires surveillance tools to detect disease-relevant strains. Whole-genome sequencing enabled detailed analysis of the capsule loci of G. parasuis [16] and the development of a multiplex serotyping PCR $[17,18]$. This PCR reaction uses specific primers based on serovar-specific variations on the capsule loci, capable of discriminating 14 of the 15 recognized G. parasuis serovars but not serovars 5 and 12. A new G. parasuis serovar-specific PCR scheme containing primers that differentiate serovars 5 and 12 was subsequently described [19]. However, the primer for serovar 12 is based on a hypothetical gene, and further investigations are warranted.

In parallel to molecular serotyping, novel PCR methods were developed to track virulence markers for G. parasuis isolates. A group of specific virulence-associated genes, named virulence-associated trimeric autotransporters $(v t a A)$ group 1-translocator, were associated with virulent G. parasuis strains [20]. Additionally, two different leader sequences were detected in the $v t a A$ genes. A test for predicting the virulence potential of $G$. parasuis strains was developed based on the leader sequence of the $v t a A$ genes, called LS-PCR [21].

Such epidemiological investigations are essential for designing preventive health programs, supporting vaccine candidate selection, improving pig flow management, and limiting the reliance on prophylactic antimicrobials. This study's objective was to characterize strains of G. parasuis from top pork-producing countries in terms of the relative geographic prevalence of molecular serovars and their relationship with $v t a A$ markers.

\section{Materials and methods}

\section{Isolate collection}

The 950 G. parasuis isolates in this collection originated from pigs from different geographical regions; USA ( $n=223$ from 15 different states), Europe $(n=436$ from 13 different Member States), China ( $n=167$ from 12 different provinces), Vietnam ( $n=56$, from two provinces), and Canada ( $n=68$, from Quebec) (Figure 1, Additional file 1). The dates of collection of isolates ranged between 2014-2018 for USA isolates, 2000-2006, 2015-2016 or unknown for European isolates, 1999-2007 or unknown for Chinese isolates, 2017 for Vietnamese isolates, and 2017-2019 for Canadian isolates (Additional file 1). The G. parasuis isolates used in this study came from diagnostic and research laboratories that are national leaders in their own countries regarding swine diseases, including Glässer's disease. Only isolates with a designated serovar were included. However, more specific information regarding the clinical history and epidemiology was not available for most isolates and was not included in this study.

Data for US isolates $(n=223)$ were obtained from the Iowa State University Veterinary Diagnostic Laboratory (ISU VDL), where isolates were confirmed as G. parasuis using Bruker matrix-assisted laser desorption time-offlight mass spectrometry (MALDI_TOF MS). Data for Canadian strains $(n=66)$ were obtained from the Diagnostic Laboratory of the Faculty of Veterinary Medicine of the University of Montreal (DLFVMUM), where isolates were also firstly identified by MALDI_TOF MS and confirmed by PCR [22]. Data for European isolates

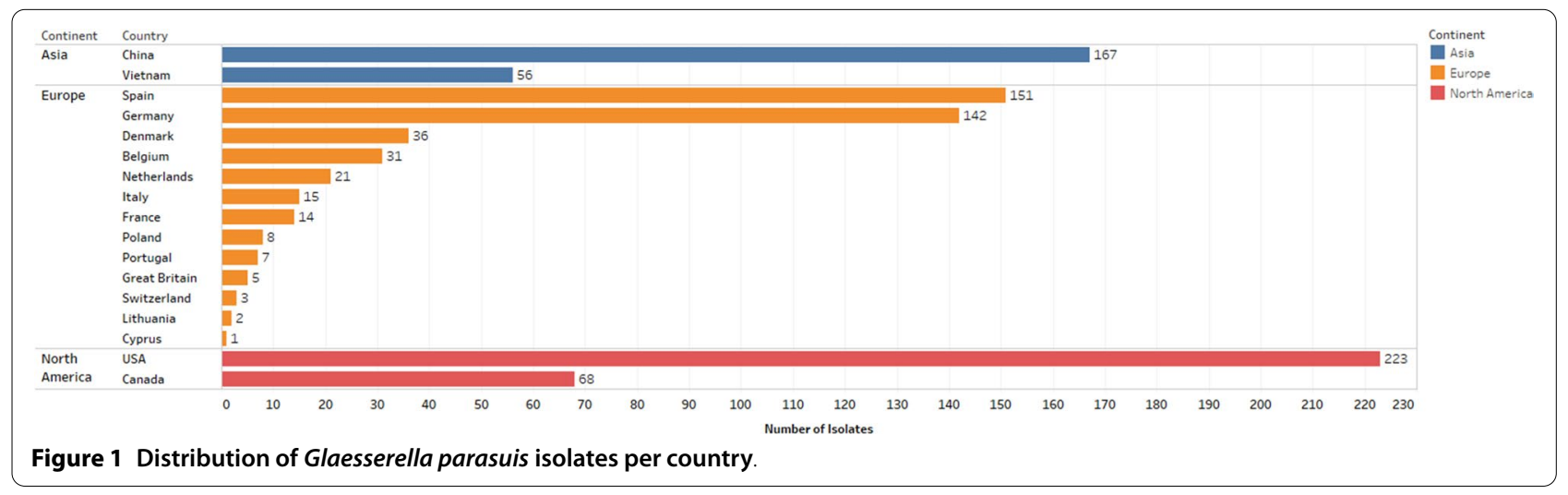


were obtained from Spain (IRTA-CReSA, $n=275$ ) and Germany (IVD Innovative Veterinary Diagnostics (IVD $\mathrm{GmbH}),(n=165)$ and represented 13 different member states. Confirmation of $G$. parasuis by both laboratories was achieved by PCR [22]. Data for Chinese isolates $(n=167)$ and Vietnamese isolates $(n=56)$ were obtained from Huazhong Agricultural University Veterinary Diagnostic Laboratory. The Chinese and Vietnamese isolates were firstly identified with biochemical tests [23] and confirmed by $16 \mathrm{~S}$ rRNA PCR [25].

Metadata for a subset of these isolates included isolation site, year of isolation, and country of origin (Additional file 1). Metadata for the strains were used to classify isolates considering clinical (internal organs) and non-clinical (URT) source when such information was available. Further classification was performed considering clinical (internal) isolates that were obtained from systemic sites or pulmonary (lungs) and non-clinical (URT) origins. G. parasuis strains isolated from internal organs other than the lungs, such as pleura, pericardium, peritoneum, joint, and meninges, were considered systemic strains. All URT strains in this study were isolated from nasal cavities.

\section{Serotyping PCR}

Serotyping PCR was undertaken by each participating laboratory with some laboratory-specific amendments. DNA extraction was achieved by standard methods; typically, a loopful of bacterial colonies were removed from growth media and suspended in $300 \mu \mathrm{L}$ of $0.01 \mathrm{M}$ sterile phosphate-buffered saline ( $\mathrm{pH}$ 7.4) without calcium and magnesium (GIBCO/Life Technologies Corporation, Carlsbad, CA, USA) and vortexed. Bacterial DNA was extracted with the MagMAX ${ }^{\mathrm{TM}}$ Viral DNA/RNA Isolation Kit (Life Technologies, Carlsbad, CA, USA) using extraction protocol per manufacturer instructions.

All participating laboratories conducted the serotyping PCR as previously described [17] with some (ISU VDL, DLFVMUM, and IVD) using the modifications made by Lacouture et al. [18].

Briefly, each PCR reaction (one for each of the primer mixtures) consisted of $12.5 \mu \mathrm{L}$ of $2 \times$ Multiplex PCR Master Mix (Qiagen, Hilden, Germany), $2.5 \mu \mathrm{L}$ of the $100 \mathrm{nmol} 1^{-1}$ of primer mix 1,2 or $3,8 \mu \mathrm{L}$ of nucleasefree water, and $2 \mu \mathrm{L}$ of extracted DNA. The reaction was run using the following conditions: first cycle at $95^{\circ} \mathrm{C}$ for $15 \mathrm{~min}$, followed by $40 \mathrm{cycles}$ of $94^{\circ} \mathrm{C}$ for $30 \mathrm{~s}, 58^{\circ} \mathrm{C}$ for $90 \mathrm{~s}$, and $72{ }^{\circ} \mathrm{C}$ for $90 \mathrm{~s}$. This was followed by one cycle at $72{ }^{\circ} \mathrm{C}$ for $10 \mathrm{~min}$. Electrophoresis and visualization of the PCR products were performed using QIAxcel Capillary Electrophoresis with screen gel software (Qiagen).

For isolates obtained by IRTA-CReSA, PCR conditions were amended using the same primers as Howell et al.
[17], but these were tested individually as follows: $94{ }^{\circ} \mathrm{C}$ for $5 \mathrm{~min}$, followed by 30 cycles of $94{ }^{\circ} \mathrm{C}$ for $30 \mathrm{~s}, 55^{\circ} \mathrm{C}$ for $30 \mathrm{~s}$, and $72{ }^{\circ} \mathrm{C}$ for $60 \mathrm{~s}$, and final extension of $72{ }^{\circ} \mathrm{C}$ for $7 \mathrm{~min}$. PCR products were visualized by agarose gel electrophoresis.

\section{Virulence gene PCR (LS-PCR)}

Isolates from North America and Europe were tested using a PCR to detect the leader sequence (LS) of the $v t a A$ genes domain as a diagnostic tool to predict G. parasuis virulence [21]. Briefly, the PCR reaction consisted of $12.5 \mu \mathrm{L}$ of $2 \times$ Multiplex PCR Master Mix (Qiagen), $1 \mu \mathrm{L}$ of the $100 \mathrm{nmol} 1^{-1}$ of each primer, $4.5 \mu \mathrm{L}$ of nuclease-free water, and $2 \mu \mathrm{L}$ of extracted DNA in a final volume of $25 \mu \mathrm{L}$. Cycling conditions were $15 \mathrm{~min}$ at $95{ }^{\circ} \mathrm{C}$, followed by 30 cycles of $45 \mathrm{~s}$ at $94{ }^{\circ} \mathrm{C}, 45 \mathrm{~s}$ at $52{ }^{\circ} \mathrm{C}$ and $1 \mathrm{~min}$ at $72{ }^{\circ} \mathrm{C}$, then a final incubation at $72{ }^{\circ} \mathrm{C}$ for $10 \mathrm{~min}$.

\section{Statistical analysis}

Associations between serovars, sample site, country of isolation, and LS-PCR results were calculated using Chisquare and Fisher's exact tests. A multinomial logistic regression model was fit to compare the sample site and serovar classification to the reference (untypeable or NT) serovar. All tests were 2 -sided, and a $p$-value of lower than 0.05 was defined as indicating a significant difference. All analyses were completed using SAS version 9.4.

\section{Results}

\section{Distribution of G. parasuis serovars}

Most of the G. parasuis isolates (886/950, 93.3\%), were typeable by serotyping PCR, and the remaining 64 (9.7\%) were identified as non-typeable (NT). All 15 serovars were identified. Serovars 4 (17.4\%), 5/12 (16.8\%), 7 (11.4\%), 1 (10.7\%), and 13 (9.1\%) were the most commonly detected serovars across the participating regions (Figure 2, Table 1, Additional files 2, 3, 4).

\section{Internal isolates}

Information on the site of origin for each isolate was available for 834 of the total 950 G. parasuis isolates, allowing classification of $G$. parasuis strains as either nasal or internal (Table 2). Internal isolates represented $81.1 \%(676 / 834)$ of all isolates for which an attributable sample site was recorded (Table 2). The most frequently identified serovars from internal locations included serovars $4,5 / 12,1,7$, and 13 in descending order, with serovars 4 and $5 / 12$ being similarly represented, at 19.2 and $18.2 \%$, respectively.

Further classification of internal isolates into pulmonary and systemic was possible for a subset of 519 of these isolates. Almost sixty percent of these further classified isolates $(307 / 519,59.2 \%)$ were obtained from 


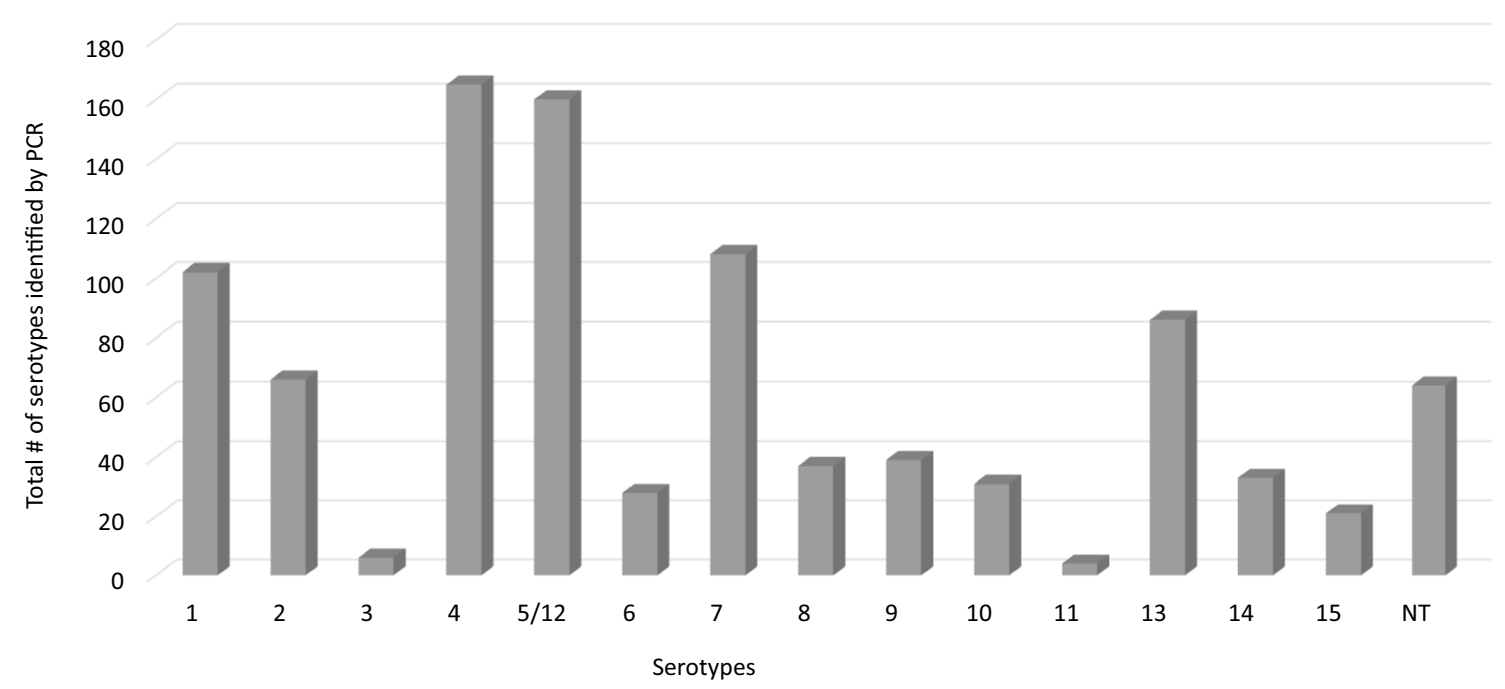

Figure 2 Frequency distribution of Glaesserella parasuis serovars identified by PCR for all of the isolates in the present study

Table 1 Number and percentage of Glaesserella parasuis serovars per country or region.

\begin{tabular}{|c|c|c|c|c|c|c|}
\hline Serovar & USA/\% & Canada/\% & Europe/\% & China/\% & Vietnam/\% & Total" \\
\hline 1 & $32 / 14.3$ & $4 / 5.9$ & $53 / 12.2$ & $12 / 7.2$ & $1 / 1.8$ & 102 \\
\hline 2 & $28 / 12.6$ & $4 / 5.9$ & $13 / 3$ & $8 / 4.8$ & $13 / 23.2$ & 66 \\
\hline 3 & $0 / 0$ & $1 / 1.5$ & $0 / 0$ & $5 / 3$ & 0/0 & 6 \\
\hline 4 & $53 / 23.8$ & $8 / 11.8$ & $75 / 17.2$ & $19 / 11.4$ & $10 / 17.9$ & 165 \\
\hline $5 / 12$ & $28 / 12.6$ & $21 / 30.9$ & $60 / 13.8$ & $36 / 21.6$ & $15 / 26.8$ & 160 \\
\hline 6 & $2 / 0.9$ & $1 / 1.5$ & $12 / 2.8$ & $12 / 7.2$ & $1 / 1.8$ & 28 \\
\hline 7 & $41 / 18.4$ & $11 / 16.2$ & $51 / 11.7$ & $4 / 2.4$ & $1 / 1.8$ & 108 \\
\hline 8 & $0 / 0$ & $2 / 2.9$ & $21 / 4.8$ & $13 / 7.8$ & $1 / 1.8$ & 37 \\
\hline 9 & $0 / 0$ & $0 / 0$ & $26 / 6$ & $7 / 4.2$ & $6 / 10.7$ & 39 \\
\hline 10 & $0 / 0$ & $0 / 0$ & $18 / 4.1$ & $6 / 3.6$ & $7 / 12.5$ & 31 \\
\hline 11 & 0/0 & 0/0 & $3 / 0.7$ & $1 / 0.6$ & $0 / 0$ & 4 \\
\hline 13 & $23 / 10.3$ & $6 / 8.8$ & $51 / 11.7$ & $6 / 3.6$ & $0 / 0$ & 86 \\
\hline 14 & $12 / 5.4$ & $2 / 2.9$ & $11 / 2.5$ & $8 / 4.8$ & $0 / 0$ & 33 \\
\hline 15 & $1 / 0.4$ & $1 / 1.5$ & $18 / 4.1$ & $1 / 0.6$ & $0 / 0$ & 21 \\
\hline NT & $3 / 1.3$ & $7 / 10.3$ & $24 / 5.5$ & 29/17.4 & $1 / 1.8$ & 64 \\
\hline Total $^{\S}$ & $223 / 100$ & $68 / 100$ & $436 / 100$ & $167 / 100$ & $56 / 100$ & 950 \\
\hline
\end{tabular}

"Sum of number of serovars in each row

$\S$ Sum of number of serovars in each column

pulmonary sites, while $40.8 \%(212 / 519)$ were of systemic origin (Table 2). Serovar 4 strains were more frequently isolated from lung samples than serovar $5 / 12$ strains (27 and $14.7 \%$, respectively). In contrast, when considering systemic isolates separately, the percentage of serovar $5 / 12$ strains was higher compared with serovar 4 (22.2 and 13.7\%, respectively) (Table 2). Likewise, both serovars 7 and 13 were more frequently detected within internal samples, than nasal samples. However, while serovar 7 was similarly represented within both pulmonary and systemic collections (12.1 and $15.6 \%$ of each collection, respectively), the percentage of serovar 13 strains was higher in the systemic collection compared with the pulmonary one (14.2 and 9.1\%, respectively) (Table 2).

Clinically relevant serovars from internal sites, such as serovars $4,5 / 12,7,1$, and 13 , were among the six serovars more frequently observed in North America and Europe, with similar distribution among systemic and pulmonary sites (Tables 3, 4, 5). Among Asian 
Table 2 Number and percentage of Glaesserella parasuis serovars per site of isolation.

\begin{tabular}{lllllr}
\hline Serovar & Nasal/\% & Internal/\% & Pulmonary*/\% & Systemic*\% & Total" \\
\hline 1 & $19 / 12$ & $75 / 11.1$ & $37 / 12.1$ & $29 / 13.7$ & 94 \\
2 & $6 / 3.8$ & $53 / 7.8$ & $31 / 10.1$ & $14 / 6.6$ & 59 \\
3 & $0 / 0$ & $6 / 0.9$ & $0 / 0$ & $1 / 0.5$ & 6 \\
4 & $18 / 11.4$ & $130 / 19.2$ & $83 / 27$ & $29 / 13.7$ & 148 \\
$5 / 12$ & $13 / 8.2$ & $124 / 18.3$ & $45 / 14.7$ & $47 / 22.2$ & 137 \\
6 & $7 / 4.4$ & $19 / 2.8$ & $2 / 0.7$ & $5 / 2.4$ & 26 \\
7 & $8 / 5.1$ & $74 / 10.9$ & $37 / 12.1$ & $33 / 15.6$ & 82 \\
8 & $19 / 12$ & $16 / 2.4$ & $3 / 1$ & $1 / 0.5$ & 35 \\
9 & $18 / 11.4$ & $17 / 2.5$ & $6 / 2$ & $4 / 1.9$ & 35 \\
10 & $15 / 9.5$ & $14 / 2.1$ & $7 / 2.3$ & $1 / 0.5$ & 29 \\
11 & $0 / 0$ & $4 / 0.6$ & $2 / 0.7$ & $1 / 0.5$ & 4 \\
13 & $8 / 5.1$ & $63 / 9.3$ & $28 / 9.1$ & $30 / 14.2$ & 71 \\
14 & $1 / 0.6$ & $30 / 4.4$ & $15 / 4.9$ & $7 / 3.3$ & 31 \\
15 & $17 / 10.8$ & $2 / 0.3$ & $0 / 0$ & $1 / 0.5$ & 19 \\
NT & $9 / 5.7$ & $49 / 7.2$ & $11 / 3.6$ & $9 / 4.2$ & 58 \\
Total $^{\S}$ & $158 / 100$ & $676 / 100$ & $307 / 100$ & $212 / 100$ & 834 \\
\hline
\end{tabular}

*Information was available for 519 internal isolates, which were divided into pulmonary and systemic

"Sum of nasal and internal isolates in each row

${ }^{\S}$ Sum of values in each column

isolates, serovar $5 / 12$ was the most prevalent, followed by serovars 4 and 2 (Table 3 ).

The highest proportion of untypable internal isolates $(18.5 \%, 29 / 157)$ was found among Chinese isolates, while only $1.4 \%(3 / 210)$ of USA internal isolates were untypable (Table 3 ).

\section{Nasal isolates}

Nasal isolates represented $18.1 \%(158 / 834)$ of all isolates for which an attributable sample site was recorded and belonged to a variety of serovars, especially serovars 8 (12\%), 1 (12\%), 4 (11.4\%), 9 (11.4\%), 15 (10.8\%) and $10(9.5 \%)$ (Table 2). While serovars 1 and 4 were more consistently found within systemic or pulmonary sites, serovars $8,9,15$, and 10 were predominantly obtained from nasal samples (Table 2).

Most of the nasal isolates in this study were obtained from Europe $(76.6 \%, 121 / 158)$ (Table 6). The most prevalent nasal serovars in Europe were 8, 15, 1, 4, 9, and 10. Most of these serovars were not prevalent among internal samples, except for serovars 1 and 4, which were also prevalent among pulmonary and systemic sites (Tables 3, 4, 5, 6).

Besides these European nasal isolates, 35 G. parasuis nasal isolates were from Asian countries, including 25 from Vietnam and ten from China. Most of the nasal isolates from China were either from serovar $5 / 12$ or 1 , while most of the nasal isolates from Vietnam were from serovars 2, 9, 4, and 10 (Tables 6). However, only serovars 1 and 9 were predominantly higher among nasal isolates. The other serovars were also frequently found among internal isolates (Table 3).

Table 3 Number and percentage of Internal Glaesserella parasuis serovars per country or region.

\begin{tabular}{|c|c|c|c|c|c|c|}
\hline Serovar & USA/\% & Canada/\% & Europe/\% & China/\% & Vietnam $/ \%$ & Totalq \\
\hline 1 & $31 / 14.8$ & $3 / 6.7$ & $32 / 13.7$ & $9 / 5.7$ & $0 / 0$ & 75 \\
\hline 2 & $27 / 12.9$ & $3 / 6.7$ & $8 / 3.4$ & $8 / 5.1$ & $7 / 22.6$ & 53 \\
\hline 3 & 0/0 & $1 / 2.2$ & 0/0 & $5 / 3.2$ & $0 / 0$ & 6 \\
\hline 4 & $51 / 24.3$ & $1 / 2.2$ & $54 / 23.2$ & $18 / 11.5$ & $6 / 19.4$ & 130 \\
\hline $5 / 12$ & 28/13.3 & $15 / 33.3$ & $35 / 15$ & $32 / 20.4$ & $14 / 45.2$ & 124 \\
\hline 6 & $2 / 1$ & $1 / 2.2$ & $4 / 1.7$ & $12 / 7.6$ & $0 / 0$ & 19 \\
\hline 7 & $34 / 16.2$ & $8 / 17.8$ & $28 / 12$ & $4 / 2.5$ & $0 / 0$ & 74 \\
\hline 8 & $0 /$ & $1 / 2.2$ & $3 / 1.3$ & $12 / 7.6$ & $0 / 0$ & 16 \\
\hline 9 & $0 / 0$ & $0 / 0$ & $10 / 4.3$ & $7 / 4.5$ & $0 / 0$ & 17 \\
\hline 10 & $0 / 0$ & 0/0 & $4 / 1.7$ & $6 / 3.8$ & $4 / 42.9$ & 14 \\
\hline 11 & 0/0 & 0/0 & $3 / 1.3$ & $1 / 0.6$ & $0 / 0$ & 4 \\
\hline 13 & $22 / 10.5$ & $4 / 8.9$ & $32 / 13.7$ & $5 / 3.2$ & $0 / 0$ & 63 \\
\hline 14 & $12 / 5.7$ & $1 / 2.2$ & $9 / 3.9$ & $8 / 5.1$ & $0 / 0$ & 30 \\
\hline 15 & 0/0 & $1 / 2.2$ & $0 / 0$ & $1 / 0.6$ & $0 / 0$ & 2 \\
\hline NT & $3 / 1.4$ & $6 / 13.3$ & $11 / 4.7$ & $29 / 18.5$ & $0 / 0$ & 49 \\
\hline Total $^{\S}$ & $210 / 100$ & $45 / 100$ & $233 / 100$ & $157 / 100$ & $31 / 100$ & 676 \\
\hline
\end{tabular}

\footnotetext{
"Sum of number of serovars in each row.
}

${ }^{\S}$ Sum of number of serovars in each column. 
Table 4 Number and percentage of Pulmonary Glaesserella parasuis serovars per country or region.

\begin{tabular}{llllr}
\hline Serovar & USA/\% & Europe/\% & Vietnam/\% & Total $^{\text {I }}$ \\
\hline 1 & $15 / 11.9$ & $22 / 14.7$ & $0 / 0$ & 37 \\
2 & $17 / 13.5$ & $7 / 4.7$ & $7 / 22.6$ & 31 \\
4 & $39 / 31$ & $38 / 25.3$ & $6 / 19.4$ & 83 \\
$5 / 12$ & $15 / 11.9$ & $16 / 10.7$ & $14 / 45.2$ & 45 \\
6 & $1 / 0.8$ & $1 / 0.7$ & $0 / 0$ & 2 \\
7 & $19 / 15.1$ & $18 / 12$ & $0 / 0$ & 37 \\
8 & $0 / 0$ & $3 / 2$ & $0 / 0$ & 3 \\
9 & $0 / 0$ & $6 / 4$ & $0 / 0$ & 6 \\
10 & $0 / 0$ & $3 / 2$ & $4 / 12.9$ & 7 \\
11 & $0 / 0$ & $2 / 1.3$ & $0 / 0$ & 2 \\
13 & $7 / 5.6$ & $21 / 14$ & $0 / 0$ & 28 \\
14 & $11 / 8.7$ & $4 / 2.7$ & $0 / 0$ & 15 \\
NT & $2 / 1.6$ & $9 / 6$ & $0 / 0$ & 11 \\
Total $^{\S}$ & $126 / 100$ & $150 / 100$ & $31 / 100$ & 307 \\
\hline
\end{tabular}

"Sum of number of serovars in each row.

${ }^{\S}$ Sum of number of serovars in each column.

There is no information whether Canadian and Chinese internal isolates originated from pulmonary sites.

Table 5 Number and percentage of Systemic Glaesserella parasuis serovars per country or region.

\begin{tabular}{|c|c|c|c|c|}
\hline Serovar & USA/\% & Canada/\% & Europe/\% & Total $^{\text {? }}$ \\
\hline 1 & 16/19 & $3 / 6.7$ & $10 / 12$ & 29 \\
\hline 2 & $10 / 11.9$ & $3 / 6.7$ & $1 / 1.2$ & 14 \\
\hline 3 & $0 / 0$ & $1 / 2.2$ & $0 / 0$ & 1 \\
\hline 4 & $12 / 14.3$ & $1 / 2.2$ & 16/19.3 & 29 \\
\hline $5 / 12$ & $13 / 15.5$ & 15/33.3 & $19 / 22.9$ & 47 \\
\hline 6 & $1 / 1.2$ & $1 / 2.2$ & $3 / 3.6$ & 5 \\
\hline 7 & $15 / 17.9$ & $8 / 17.8$ & $10 / 12$ & 33 \\
\hline 8 & $0 / 0$ & $1 / 2.2$ & $0 / 0$ & 1 \\
\hline 9 & $0 / 0$ & $0 / 0$ & $4 / 4.8$ & 4 \\
\hline 10 & $0 / 0$ & $0 / 0$ & $1 / 1.2$ & 1 \\
\hline 11 & $0 / 0$ & $0 / 0$ & $1 / 1.2$ & 1 \\
\hline 13 & $15 / 17.9$ & $4 / 8.9$ & 11/13.3 & 30 \\
\hline 14 & $1 / 1.2$ & $1 / 2.2$ & $5 / 6$ & 7 \\
\hline 15 & $0 / 0$ & $1 / 2.2$ & $0 / 0$ & 1 \\
\hline NT & $1 / 1.2$ & 6/13.3 & $2 / 2.4$ & 9 \\
\hline Total ${ }^{\S}$ & $84 / 100$ & $45 / 100$ & $83 / 100$ & 212 \\
\hline
\end{tabular}

"Sum of number of serovars in each row.

$\S$ Sum of number of serovars in each column.

There is no information whether Asian internal isolates originated from systemic sites.
Table 6 Number and percentage of Nasal Glaesserella parasuis serovars per country or region.

\begin{tabular}{lllllr}
\hline Serovar & USA/\% & Europe/\% & China/\% & Vietnam/\% & Total" $^{\text {I }}$ \\
\hline 1 & $1 / 50$ & $14 / 11.6$ & $3 / 30$ & $1 / 4$ & 19 \\
2 & $0 / 0$ & $0 / 0$ & $0 / 0$ & $6 / 24$ & 6 \\
4 & $1 / 50$ & $12 / 9.9$ & $1 / 10$ & $4 / 16$ & 18 \\
$5 / 12$ & $0 / 0$ & $8 / 6.6$ & $4 / 40$ & $1 / 4$ & 13 \\
6 & $0 / 0$ & $6 / 5$ & $0 / 0$ & $1 / 4$ & 7 \\
7 & $0 / 0$ & $7 / 5.8$ & $0 / 0$ & $1 / 4$ & 8 \\
8 & $0 / 0$ & $17 / 14$ & $1 / 10$ & $1 / 4$ & 19 \\
9 & $0 / 0$ & $12 / 9.9$ & $0 / 0$ & $6 / 24$ & 18 \\
10 & $0 / 0$ & $12 / 9.9$ & $0 / 0$ & $3 / 12$ & 15 \\
13 & $0 / 0$ & $7 / 5.8$ & $1 / 10$ & $0 / 0$ & 8 \\
14 & $0 / 0$ & $1 / 0.8$ & $0 / 0$ & $0 / 0$ & 1 \\
15 & $0 / 0$ & $17 / 14$ & $0 / 0$ & $0 / 0$ & 17 \\
NT & $0 / 0$ & $8 / 6.6$ & $0 / 0$ & $1 / 4$ & 9 \\
Total $^{5}$ & $2 / 100$ & $121 / 100$ & $10 / 100$ & $25 / 100$ & 158 \\
\hline
\end{tabular}

"Sum of number of serovars in each row.

$\S$ Sum of number of serovars in each column.

There were no nasal G. parasuis isolates submitted from Canada.

\section{Association between Glaesserella parasuis serovars and site of isolation}

Results of the chi-square analysis showed an association between sample type (nasal/internal) and serovar $(p<0.001)$. A multinomial logistic regression model was further used to predict serovar classification based on sample type. Given the sample type (nasal/internal), samples collected from the nasal cavity had a higher probability of being of serovars $8,9,10$, or $15(p<0.05)$, but no association was found between serovar and internal samples, during this initial analysis.

Using the same model, internal isolates were classified as either pulmonary or systemic and compared against nasal isolates for the subsequent analysis. Given the sample type, systemic isolates had a higher probability of being of serovar $5 / 12,13$, or 7 compared to the nasal sample collection $(p<0.01)$. In comparison, pulmonary isolates had a higher likelihood of being serovars $2,4,7$, or $14(p<0.01)$. No statistical significance was observed when comparing systemic and pulmonary isolates. These results agree with Olvera et al. [20], where pulmonary isolates could be either virulent or non-virulent since the lung can be populated by upper respiratory tract commensal microbiota and strains that harbor certain virulence factors that enable them to survive the lung defenses and invade systemically. As shown in Table 2, $14 / 53$ and $7 / 31$ of serovars 2 and 14, respectively, were also found on systemic sites. 


\section{Association between Glaesserella parasuis serovars and LS-PCR result}

G. parasuis isolates were classified as potentially virulent based on the presence or absence of specific leader sequences within their $v t a A$ genes, using multiplex LSPCR. Within this G. parasuis collection, 727 isolates were tested by LS-PCR among North American and European isolates (Table 7). Overall, 85.7\% (623/727) of the G. parasuis isolates were classified as virulent by LS-PCR, $14.3 \%(104 / 727)$ were classified as non-virulent by LSPCR, and 223 isolates were not tested (Table 7).

Most of the disease-associated isolates, including 1, 2, $4,5 / 12,7,13$, and 14, were considered virulent by LSPCR, with few exceptions where these virulent serovars were deemed to be non-virulent by LS-PCR (10/578) (Table 7). In contrast, most of the isolates from the nasal cavity, including serovars $6,9,10$, and 15 , were considered non-virulent by LS-PCR (Table 8), with a significant association between serovar and classification as virulent or non-virulent by LS-PCR $(p<0.001)$. Further analysis was performed within each serovar to evaluate the association of virulent and non-virulent strains with the site of isolation. A significant association was found for serovars 5/12 and 7, which had a higher chance of being virulent and from internal sites (systemic or pulmonary). Contrarily, serovar 10 had a higher chance of being nonvirulent and from a nasal site (Table 8).

Table 7 Number and percentage of virulent and nonvirulent isolates within the same serovar.

\begin{tabular}{|c|c|c|c|c|}
\hline Serovar & $\begin{array}{l}\text { Virulent by } \\
\text { LS-PCR/\% }\end{array}$ & $\begin{array}{l}\text { Non-Virulent } \\
\text { by LS-PCR/\% }\end{array}$ & $\begin{array}{l}\text { Not Tested by } \\
\text { LS-PCR/\% }\end{array}$ & Total $^{\text {? }}$ \\
\hline 1 & $89 / 87.3$ & $0 / 0$ & $13 / 12.7$ & 102 \\
\hline 2 & $44 / 66.7$ & $1 / 1.5$ & $21 / 31.8$ & 66 \\
\hline 3 & $0 / 0$ & $1 / 16.7$ & 5/83.3 & 6 \\
\hline 4 & $136 / 82.4$ & $0 / 0$ & 29/17.6 & 165 \\
\hline $5 / 12$ & $106 / 66.3$ & $3 / 1.9$ & $51 / 31.9$ & 160 \\
\hline 6 & 4/14.3 & $11 / 39.3$ & $13 / 46.4$ & 28 \\
\hline 7 & $101 / 93.5$ & $2 / 1.9$ & $5 / 4.6$ & 108 \\
\hline 8 & $0 / 0$ & $23 / 62.2$ & $14 / 37.8$ & 37 \\
\hline 9 & $6 / 15.4$ & $20 / 51.3$ & $13 / 33.3$ & 39 \\
\hline 10 & $2 / 6.5$ & 16/51.6 & $13 / 41.9$ & 31 \\
\hline 11 & $3 / 75$ & $0 / 0$ & $1 / 25$ & 4 \\
\hline 13 & $77 / 89.5$ & $3 / 3.5$ & $6 / 7$ & 86 \\
\hline 14 & $25 / 75.8$ & $0 / 0$ & $8 / 24.2$ & 33 \\
\hline 15 & $3 / 14.3$ & $17 / 81$ & $1 / 4.8$ & 21 \\
\hline NT & $27 / 42.2$ & 7/10.9 & $30 / 46.9$ & 64 \\
\hline Total $\left.\right|^{\S}$ & $623 / 65.6$ & $104 / 10.9$ & $223 / 23.5$ & 950 \\
\hline
\end{tabular}

"Sum of number of serovars in each row.

$\S$ Sum of number of serovars in each column.
Table 8 Distribution of Glaesserella parasuis serovars by site of isolation and LS-PCR results.

\begin{tabular}{|c|c|c|c|c|c|}
\hline & LS-PCR & Systemic & Pulmonary & Nasal & $P$-value \\
\hline \multirow[t]{2}{*}{ Serovar 1} & Virulent & 29 & 37 & 15 & \\
\hline & $\begin{array}{l}\text { Non-Viru- } \\
\text { lent }\end{array}$ & 0 & 0 & 0 & Not Tested* \\
\hline \multirow[t]{2}{*}{ Serovar 2} & Virulent & 14 & 23 & 0 & \\
\hline & $\begin{array}{l}\text { Non-Viru- } \\
\text { lent }\end{array}$ & 0 & 1 & 0 & 0.439 \\
\hline \multirow[t]{2}{*}{ Serovar 3} & Virulent & 0 & 0 & 0 & \\
\hline & $\begin{array}{l}\text { Non-Viru- } \\
\text { lent }\end{array}$ & 1 & 0 & 0 & Not Tested \\
\hline \multirow[t]{2}{*}{ Serovar 4} & Virulent & 14 & 23 & 0 & \\
\hline & $\begin{array}{l}\text { Non-Viru- } \\
\text { lent }\end{array}$ & 0 & 1 & 0 & 0.439 \\
\hline \multirow{2}{*}{$\begin{array}{c}\text { Serovar } \\
5 / 12\end{array}$} & Virulent & 47 & 30 & 6 & \\
\hline & $\begin{array}{l}\text { Non-Viru- } \\
\text { lent }\end{array}$ & 0 & 1 & 2 & 0.002 \\
\hline \multirow[t]{2}{*}{ Serovar 6} & Virulent & 1 & 0 & 2 & \\
\hline & $\begin{array}{l}\text { Non-Viru- } \\
\text { lent }\end{array}$ & 4 & 2 & 4 & 0.612 \\
\hline \multirow[t]{2}{*}{ Serovar 7} & Virulent & 33 & 37 & 5 & \\
\hline & $\begin{array}{l}\text { Non-Viru- } \\
\text { lent }\end{array}$ & 0 & 0 & 2 & 0.000 \\
\hline \multirow[t]{2}{*}{ Serovar 8} & Virulent & 0 & 0 & 0 & \\
\hline & $\begin{array}{l}\text { Non-Viru- } \\
\text { lent }\end{array}$ & 1 & 3 & 17 & Not Tested \\
\hline \multirow[t]{2}{*}{ Serovar 9} & Virulent & 0 & 3 & 1 & \\
\hline & $\begin{array}{l}\text { Non-Viru- } \\
\text { lent }\end{array}$ & 4 & 3 & 11 & 0.056 \\
\hline \multirow[t]{2}{*}{ Serovar 10} & Virulent & 1 & 1 & 0 & \\
\hline & $\begin{array}{l}\text { Non-Viru- } \\
\text { lent }\end{array}$ & 0 & 2 & 12 & 0.007 \\
\hline \multirow[t]{2}{*}{ Serovar 11} & Virulent & 1 & 2 & 0 & \\
\hline & $\begin{array}{l}\text { Non-Viru- } \\
\text { lent }\end{array}$ & 0 & 0 & 0 & Not Tested \\
\hline \multirow[t]{2}{*}{ Serovar 13} & Virulent & 30 & 26 & 6 & \\
\hline & $\begin{array}{l}\text { Non-Viru- } \\
\text { lent }\end{array}$ & 0 & 2 & 1 & 0.188 \\
\hline \multirow[t]{2}{*}{ Serovar 14} & Virulent & 7 & 15 & 1 & \\
\hline & $\begin{array}{l}\text { Non-Viru- } \\
\text { lent }\end{array}$ & 0 & 0 & 0 & Not Tested \\
\hline \multirow[t]{2}{*}{ Serovar 15} & Virulent & 0 & 0 & 2 & \\
\hline & $\begin{array}{l}\text { Non-Viru- } \\
\text { lent }\end{array}$ & 1 & 0 & 15 & 0.716 \\
\hline
\end{tabular}

*Not Tested: All isolates from this serovar had the same LS-PCR result.

$P$-value $<0.05$ was considered significant.

\section{Discussion}

Serotyping has been a necessary means of characterizing G. parasuis isolates. Using a novel serotyping PCR method [17, 18], we described the distribution of G. parasuis serovars globally and within a geographic region. The data collected in this study are from different 
diagnostic laboratories and research centers. Even though they serve as centers of expertise on the diagnosis of swine diseases, the dataset used in this study is limited to isolates available in these laboratories and, therefore, do not represent a true prevalence of G. parasuis serovars. Additionally, some of the strains from Europe (57/436) and China (167/223) were isolated more than ten years ago or have an unknown date of isolation. Thus, the actual distribution (prevalence) of G. parasuis serovars is still unknown. Even though our data do not represent prevalence, most of these isolates originate from field cases and reflect strains frequently found in diseaseassociated cases, and thus useful information for swine practitioners and researchers.

The serovars' overall distribution varied slightly among countries, and four serovars $(1,4,5 / 12$, and 7$)$ accounted for half of all isolates. Serovars 4 and 5/12, in particular, were consistently represented across all regions in this study and previous studies [13, 22-28].

The serovars of utmost clinical significance were identified in samples from internal sites (serovars 4, 5/12, 1, 7, and 13) in this study. These serovars have been previously considered disease-associated [12, 13, 22-30]. Variable sample sizes limited our ability to discern significant differences in serovar prevalence at the country level. The relative scarcity of serovar 4 isolates among Canada's internal samples was notable, and serovars 1,7 , and 13 appeared relatively similarly scarce among internal samples from China and Vietnam.

An important finding in this present study and other recent studies [28-30] was that serovar 7 is a significant disease-associated serovar of G. parasuis. In this study, serovar 7 was assigned to $10.9 \%$ of all internal samples (ranked $4^{\text {th }}$ most prevalent serovar) and $15.6 \%$ of all systemic isolates (ranked second most prevalent serovar). Serovar 7 was initially found non-virulent by intraperitoneal challenge [12], and it was the most prevalent serovar identified from the URT of healthy pigs in China [14]. Conversely, a serovar 7 field strain was able to cause disease in one snatch-farrowed colostrum-deprived pig when inoculated intranasally and was consistently isolated from pulmonary and systemic locations from pigs affected by Glasser's disease in Germany [28]. Additionally, the serovar 7 reference strain 174 caused disease in colostrum-deprived and commercial pigs after experimental inoculation $[29,30]$, demonstrating this serovar's pathogenic potential [31].

Based on their detection in fewer than $1 \%$ of all internal samples, the least clinical significance serovars were serovars 3,11 , and 15 . Serovars 3 and 11 were previously considered non-virulent [12]. Still, the apparent limited clinical significance of serovar 15 contradicted previous findings [12], highlighting the limitations of small-scale challenge studies as indicators of virulence. Isolates of serovar 10 have been reported as disease and non-disease associated [31]. Still, while the present study found serovar 10 in $9.9 \%$ of nasal samples from Europe (the region supplying the most significant bulk of nasal samples), it was found in only $1.7 \%$ of internal samples from Europe along with a small cluster of internal isolates from China and Vietnam. Thus, the present study indicates only limited significance for this serovar.

Nevertheless, pigs harbor various G. parasuis strains in their URT, and potentially pathogenic serovars can be isolated from the URT of healthy pigs $[9,14]$. In this study, URT isolates originated mainly from Europe and Asia, representing $16.6 \%$ of this study's collection. Serovars $6,8,9,10$, and 15 were amongst the most commonly identified (43.6\%) in the URT. In comparison, these serovars represented only $7.2 \%$ of all internal isolates. Serovars 10 and 15 have been traditionally considered virulent, even though they are not prevalent as diseaseassociated serovars worldwide, and were classified as non-virulent by the LS-PCR. Interestingly, serovars 6 and 8 Asian isolates were also frequently isolated from Chinese samples' internal sites, representing an increase of more virulent strains belonging to these serovars in Asia.

Different pathogenic properties have also been described for G. parasuis serovar 4 strains. The reference isolate for serovar 4 was initially isolated from the nose of a healthy pig and considered mildly virulent [12]. In our study, serovar 4 isolates were mainly associated with pulmonary sites $(83 / 130,64 \%)$, followed by systemic (29/130) and nasal sites (18/130). In an earlier study, North American isolates of serovar 4 were mainly isolated from lung samples of pigs with pneumonia and other systemic sites, but not from URT samples [22]. When two serovar 4 European field isolates were used to infect pigs experimentally, one could reproduce Glasser's disease, while the other could not [31]. These results are consistent with other reports showing serovar 4 isolates clustering with virulent and non-virulent genotypes [3133]. These findings illustrate how isolates of the same serovar can have different degrees of virulence. Additionally, some G. parasuis serovars' virulence may have been wrongly assigned by the Kielstein and Rapp-Gabrielson scheme [12].

Glasser's disease is a complex multifactorial disease, and several different putative virulence genes have been identified [34-37]. Still, little experimental data is available to correlate these virulence genes with clinical disease. However, empirical evidence exists, demonstrating the group $1 v t a A$ genes' role in G. parasuis virulence [21, $38,39]$. Therefore, to help identify virulent G. parasuis 
strains, a new PCR, based on the leader sequence of the $v$ taA genes, was developed [21].

In the present study, most internal isolates were identified as virulent by LS-PCR, while most nasal isolates were non-virulent. Our results agree with previous reports [25, 28, 39-41] and supported our disease association observations for serovar 7 isolates. In our study, most serovar $7(75 / 77,97.4 \%)$ strains were internal and classified as virulent by the LS-PCR. Only two serovar 7 LS-PCR negatives were found in the collection, and these were from nasal samples. In vitro analysis was not performed in this study to further characterize the virulence of the strains. Previous genome analysis performed on a serovar 7 reference strain classified as virulent by LS-PCR, but susceptible to both phagocytosis and serum demonstrated a reduction in the number of $v \operatorname{taAs}$ in that strain, which may represent a strain with an intermediate phenotype [21].

Nevertheless, some discordant results were also found within the LS-PCR data. We found that a small number of isolates from typically non-disease-associated serovars (such as 6, 8, and 9), and classified as non-virulent by LS-PCR, were recovered from systemic sites. In this case, other environmental factors or coinfections could be responsible for creating the necessary conditions for less virulent strains to invade systemically $[21,31,40]$. Even though $v t a A$ genes are positively correlated with $G$. parasuis virulent strains and can be used as a predictor of virulence, other factors may also be necessary for disease development [25, 32, 40].

In conclusion, the overall distribution of G. parasuis serovars remains predominantly constant over the past 20 years, with few serovars representing most of the strains isolated from Glasser's disease-affected pigs and noting the confirmation of serovar 7 as a globally relevant serovar. Even though the serovar and virulence relationship is not definitive, serotyping is still suitable for the epidemiological characterization of G. parasuis in pig farms, helping implement more adequate control measures, such as selecting isolates to be included in vaccines. When serotyping is not available, the site of isolation can become crucial for screening diseaseassociated isolates since systemic strains are likely the ones that possess the necessary virulent mechanisms for disease development. To date, vtaA genes are the most well-characterized G. parasuis virulence factors. This survey of global G. parasuis isolates confirms that the LS-PCR is useful for G. parasuis virulence prediction.

\section{Supplementary Information}

The online version contains supplementary material available at https://doi. org/10.1186/s13567-021-00935-9.

Additional file 1. Metadata of Glaesserella parasuis isolates including isolation site, year of isolation, and country of origin.

Additional file 2. Distribution of Glaesserella parasuis serovars in North America.

Additional file 3. Distribution of Glaesserella parasuis serovars in Europe.

Additional file 4. Distribution of Glaesserella parasuis serovars in Asia.

\section{Acknowledgements}

We would like to acknowledge the lowa State University Diagnostic Laboratory for providing isolates and assistance with testing development. We thank Dr Xuwang Cai (Huazhong Agricultural University) for providing the Chinese clinical isolates, and Nuria Galofre-Mila and Catalina Cruañas-Paniker for their assistance with testing at the IRTA-CReSA.

\section{Authors' contributions}

All authors participated in collecting the data, performing the tests, and analyzing the data. NM, MJC, MG, KSM, AT, and VA designed the study, analyzed the data and drafted the manuscript. All authors read and approved the final manuscript.

\section{Availability of data and materials}

All data generated or analyzed during this study are included in this published article and its supplementary information files.

\section{Declarations}

Competing interests

The authors declare that they have no competing interests.

\section{Author details}

${ }^{1}$ Veterinary Diagnostic Laboratory, lowa State University, Ames, IA, USA. ${ }^{2}$ Faculty of Veterinary Medicine, University of Montreal, Montreal, Canada. ${ }^{3}$ Innovative Veterinary Diagnostics (IVD GmbH), Albert-Einstein-Str. 5, 30926 Seelze, Germany. ${ }^{4}$ Faculty of Animal Science and Veterinary Medicine, University of Agricultural and Forestry, Hue University, Hue 53000, Vietnam. ${ }^{5}$ State Key Laboratory of Agricultural Microbiology, Huazhong Agricultural University College of Veterinary Medicine, Wuhan 430070, China. ${ }^{6}$ Department of Veterinary Clinic and Surgery, Federal University of Minas Gerais, Av. Antônio Carlos, 6627, Belo Horizonte, MG, Brazil. ${ }^{7}$ PIC North America, Hendersonville, TN, USA. ${ }^{8}$ Department of Veterinary Medicine, University of Cambridge, Madingley Road, Cambridge CB3 OES, UK. ${ }^{9}$ IRTA, Centre de Recerca en Sanitat Animal (CReSA, IRTA-UAB), Campus de la Universitat Autònoma de Barcelona, 08193 Bellaterra, Spain. ${ }^{10}$ OIE Collaborating Centre for the Research and Control of Emerging and Re-Emerging Swine Diseases in Europe (IRTA-CReSA), Bellaterra, Barcelona, Spain.

Received: 24 December 2020 Accepted: 13 April 2021

Published online: 12 May 2021

\section{References}

1. Aragon V, Segales J, Tucker AW (2019) Glässer's disease. In: Zimmerman JJ, Karriker LA, Ramirez A, Schwartz K, Stevenson GW, Zhang J (eds) Diseases of Swine. Ames, Wiley-Blackwell 
2. Zhang B, Tang C, Liao M, Yue H (2014) Update on the pathogenesis of Haemophilus parasuis infection and virulence factors. Vet Microbiol 168:1-7

3. Martín de la Fuente AM, Tucker AW, Navas J, Blanco M, Morris SJ, GutiérrezMartín CB (2007) Antimicrobial susceptibility patterns of Haemophilus parasuis from pigs in the United Kingdom and Spain. Vet Microbiol 120:184-191

4. Moleres J, Santos-López A, Lázaro I, Labairu J, Prat C, Ardanuy C, GonzálezZorn B, Aragon V, Garmendia J (2015) Novel blaROB-1-bearing plasmid conferring resistance to $\beta$-lactams in Haemophilus parasuis isolates from healthy weaning pigs. Appl Environ Microbiol 81:3255-3267

5. Brogden S, Pavlović A, Tegeler R, Kaspar H, De Vaan N, Kehrenberg C (2018) Antimicrobial susceptibility of Haemophilus parasuis isolates from Germany by use of a proposed standard method for harmonized testing. Vet Microbiol 217:32-35

6. Zhang B, Ku X, Yu X, Sun Q, Wu H, Chen F, Zhang X, Guo L, Tang X, He Q (2019) Prevalence and antimicrobial susceptibilities of bacterial pathogens in Chinese pig farms from 2013 to 2017. Sci Rep 9:9908

7. Miniats OP, Smart NL, Rosendal S (1991) Cross protection among Haemophilus parasuis strains in immunized gnotobiotic pigs. Can J Vet Res 55:37-41

8. Takahashi K, Naga S, Yagihashi T, Ikehata T, Nakano Y, Senna K, Maruyama T, Murofushi J (2001) A cross-protection experiment in pigs vaccinated with Haemophilus parasuis serovars 2 and 5 bacterins, and evaluation of a bivalent vaccine under laboratory and field conditions. J Vet Med Sci 63:487-491

9. Cerdà-Cuéllar M, Naranjo JF, Verge A, Nofrarías M, Cortey M, Olvera A, Segalés J, Aragon V (2010) Sow vaccination modulates the colonization of piglets by Haemophilus parasuis. Vet Microbiol 145:315-320

10. Palzer A, Ritzmann M, Wolf G, Heinritzi K (2008) Associations between pathogens in healthy pigs and pigs with pneumonia. Vet Rec. https://doi. org/10.1136/vr.162.9.267

11. Li J, Wang S, Li C, Wang C, Liu Y, Wang G, He X, Hu L, Liu Y, Cui M, Bi C, Shao Z, Wang X, Xiong T, Cai X, Huang L, Weng C (2017) Secondary Haemophilus parasuis infection enhances highly pathogenic porcine reproductive and respiratory syndrome virus (HP-PRRSV) infection-mediated inflammatory responses. Vet Microbiol 204:35-42

12. Kielstein P, Rapp-Gabrielson VJ (1992) Designation of 15 serovars of Haemophilus parasuis on the basis of immunodiffusion using heat-stable antigen extracts. J Clin Microbiol 30:862-865

13. Angen O, Svensmark B, Mittal KR (2004) Serological characterization of Danish Haemophilus parasuis isolates. Vet Microbiol 103:255-258

14. Zhang P, Zhang C, Aragon V, Zhou X, Zou M, Wu C, Shen Z (2019) Investigation of Haemophilus parasuis from healthy pigs in China. Vet Microbiol 231:40-44

15. Mahmmod YS, Correa-Fiz F, Aragon V (2020) Variations in association of nasal microbiota with virulent and non-virulent strains of Glaesserella (Haemophilus) parasuis in weaning piglets. Vet Res 51:7

16. Howell KJ, Weinert LA, Luan SL, Peters SE, Chaudhuri RR, Harris D, Angen $\varnothing$, Aragon V, Parkhill J, Langford PR, Rycroft AN (2013) Gene content and diversity of the loci encoding biosynthesis of capsular polysaccharides of the 15 serovar reference strains of Haemophilus parasuis. J Bacteriol 195:4264-4273

17. Howell KJ, Peters SE, Wang J, Hernandez-Garcia J, Weinert LA, Luan SL, Chaudhuri RR, Angen $\varnothing$, Aragon V, Williamson SM, Parkhill J, Langford PR, Rycroft AN, Wren BW, Maskell DJ, Tucker AW (2015) Development of a multiplex PCR assay for rapid molecular serotyping of Haemophilus parasuis. J Clin Microbiol 53:3812-3821

18. Lacouture S, Rodriguez E, Strutzberg-Minder K, Gottschalk M (2017) Serotyping of Haemophilus parasuis field isolates from diseased pigs in Quebec by indirect hemagglutination assay and multiplex polymerase chain reaction (PCR). Can Vet J 58:802-804

19. Jia A, Zhou R, Fan H, Yang K, Zhang J, Xu Y, Wang G, Liao M (2017) Development of serotype-specific PCR assays for typing of Haemophilus parasuis isolates circulating in southern China. J Clin Microbiol 55:3249-3257

20. Olvera A, Pina S, Macedo N, Oliveira S, Aragon V, Bensaid A (2012) Identification of potentially virulent strains of Haemophilus parasuis using a multiplex PCR for virulence-associated autotransporters (vtaA). Vet J 191:213-218

21. Galofré-Milà N, Correa-Fiz F, Lacouture S, Gottschalk M, Strutzberg-Minder K, Bensaid A, Pina-Pedrero S, Aragon V (2017) A robust PCR for the differentiation of potential virulent strains of Haemophilus parasuis. BMC Vet Res 13:124
22. Oliveira S, Blackall PJ, Pijoan C (2003) Characterization of the diversity of Haemophilus parasuis field isolates by use of serotyping and genotyping. Am J Vet Res 64:435-442

23. Cai X, Chen H, Blackall PJ, Yin Z, Wang L, Liu Z, Jin M (2005) Serological characterization of Haemophilus parasuis isolates from China. Vet Microbiol 111:231-236

24. Rúbies X, Kielstein P, Costa L, Riera P, Artigas C, Espuña E (1999) Prevalence of Haemophilus parasuis serovars isolated in Spain from 1993 to 1997. Vet Microbiol 66:245-248

25. Van CN, Thanh TVT, Zou G, Jia M, Wang Q, Zhang L, Ding W, Huang Q, Zhou R (2019) Characterization of serotypes and virulence genes of Haemophilus parasuis isolates from Central Vietnam. Vet Microbiol 230:117-122

26. Dijkman R, Wellenberg GJ, van der Heijden HM, Peerboom R, Olvera A, Rothkamp A, Peperkamp K, van Esch EJ (2012) Analyses of Dutch Haemophilus parasuis isolates by serotyping, genotyping by ERIC-PCR and Hsp60 sequences and the presence of the virulence associated trimeric autotransporters marker. Res Vet Sci 93:589-595

27. Ma L, Wang L, Chu Y, Li X, Cui Y, Chen S, Zhou J, Li C, Lu Z, Liu J, Liu Y (2016) Characterization of Chinese Haemophilus parasuis isolates by traditional serotyping and molecular serotyping methods. PLoS One 11:e0168903

28. Schuwerk L, Hoeltig D, Waldmann KH, Strutzberg-Minder K, Valentin-Weigand P, Rohde J (2020) Serotyping and pathotyping of Glaesserella parasuis isolated 2012-2019 in Germany comparing different PCR-based methods. Vet Res 51:137

29. Dazzi C, Guizzo J, Prigol S, Kreutz L, Driemeier D, Chaudhuri S, Schryvers A, Frandoloso R (2020) New pathological lesions developed in pigs by a "Nonvirulent" strain of Glaesserella parasuis. Front Vet Sci 7:98

30. Frandoloso R, Chaudhuri S, Frandoloso G, Yu R, Schryvers A (2020) Proof of concept for prevention of natural colonization by oral needle-free administration of a microparticle vaccine. Front Immunol 11:595320

31. Aragon V, Cerdà-Cuéllar M, Fraile L, Mombarg M, Nofrarías M, Olvera A, Sibila M, Solanes D, Segalés J (2010) Correlation between clinico-pathological outcome and typing of Haemophilus parasuis field strains. Vet Microbiol 142:387-393

32. Boerlin P, Poljak Z, Gallant J, Chalmers G, Nicholson V, Soltes GA, Maclnnes II (2013) Genetic diversity of Haemophilus parasuis from sick and healthy pigs. Vet Microbiol 167:459-467

33. Wang L, Ma L, Liu Y, Gao P, Li Y, Li X, Liu Y (2016) Multilocus sequence typing and virulence analysis of Haemophilus parasuis strains isolated in five provinces of China. Infect Genet Evol 44:228-233

34. Sack M, Baltes N (2009) Identification of novel potential virulence-associated factors in Haemophilus parasuis. Vet Microbiol 136:382-386

35. Zhang J, Xu C, Guo L, Shen H, Deng X, Ke C, Ke B, Zhang B, Li A, Ren T, Liao M (2012) Prevalence and characterization of genotypic diversity of Haemophilus parasuis isolates from southern China. Can J Vet Res 76:224-229

36. Zhang NZ, Chu YF, Gao PC, Zhao P, He Y, Lu ZX (2012) Immunological identification and characterization of extracellular serine protease-like protein encoded in a putative espP2 gene of Haemophilus parasuis. J Vet Med Sci 74:983-987

37. Martínez-Moliner V, Soler-Llorens P, Moleres J, Garmendia J, Aragon V (2012) Distribution of genes involved in sialic acid utilization in strains of Haemophilus parasuis. Microbiology 158:2117-2124

38. Olvera A, Pina S, Pérez-Simó M, Oliveira S, Bensaid A (2010) Virulenceassociated trimeric autotransporters of Haemophilus parasuis are antigenic proteins expressed in vivo. Vet Res 41:26

39. Costa-Hurtado M, Ballester M, Galofré-Milà N, Darji A, Aragon V (2012) VtaA8 and VtaA9 from Haemophilus parasuis delay phagocytosis by alveolar macrophages. Vet Res 43:57

40. Turni C, Singh R, Blackall PJ (2018) Virulence-associated gene profiling, DNA fingerprinting and multilocus sequence typing of Haemophilus parasuis isolates in Australia. Aust Vet J 96:196-202

41. Lin WH, Shih HC, Lin CF, Yang CY, Chang YF, Lin CN, Chiou MT (2018) Molecular serotyping of Haemophilus parasuis isolated from diseased pigs and the relationship between serovars and pathological patterns in Taiwan. PeerJ 6:e6017

\section{Publisher's Note}

Springer Nature remains neutral with regard to jurisdictional claims in published maps and institutional affiliations. 